\title{
Probing $d$-wave pairing correlations in the pseudogap regime of the cuprate superconductors via low-energy states near impurities
}

\author{
Daniel E. Sheehy, ${ }^{*}$ Inanç Adagideli, ${ }^{\dagger}$ Paul M. Goldbart, ${ }^{\dagger}$ and Ali Yazdani ${ }^{\S}$ \\ Department of Physics and Materials Research Laboratory, University of Illinois at Urbana-Champaign, Urbana, Illinois 61801
}

(Received 1 June 2001; published 21 November 2001)

\begin{abstract}
The issue of probing the pseudogap regime of the cuprate superconductors, specifically with regard to the existence and nature of superconducting pairing correlations of $d$-wave symmetry, is explored theoretically. It is shown that if the $d$-wave correlations believed to describe the superconducting state persist into the pseudogap regime, but with pair-potential phase fluctuations that destroy their long-range nature, then the low-energy quasiparticle states observed near extended impurities in the truly superconducting state should also persist as resonances in the pseudogap regime. The scattering of quasiparticles by these phase-fluctuations broadens what was (in the superconducting state) a sharp peak in the single-particle spectral function at low energy, as we demonstrate within the context of a simple model. This peak and its broadening are, in principle, accessible via scanning tunneling spectroscopy near extended impurities in the pseudogap regime. If so, such experiments would provide a probe of the extent to which $d$-wave superconducting correlations persist upon entering the pseudogap regime, thus providing a stringent diagnostic of the phase-fluctuation scenario.
\end{abstract}

DOI: $10.1103 /$ PhysRevB.64.224518

PACS number(s): 74.25.-q, 74.40.+k, 74.72.- h

\section{INTRODUCTION}

Among the challenges presented by the high-temperature superconductors, one of the most persistent concerns the anomalous properties of the normal state of these materials. In particular, the suppression of single-particle spectral weight around the Fermi energy ${ }^{1-3}$ for temperatures above the superconducting transition temperature $T_{\mathrm{c}}$ of the underdoped cuprates indicates that the electronic behavior of these materials deviates substantially from that of conventional superconductors. There have been several theoretical scenarios proposed to explain this so-called pseudogap behavior. ${ }^{4-9}$ In the present paper, we shall be concerned with a particular one of these, viz., the phase-fluctuation scenario. ${ }^{10-13}$ According to this scenario, superconducting correlations in the form of Cooper pairing are presumed to exist (and to be responsible for the loss of single-particle spectral weight) for temperatures $T$ below the pseudogap onset temperature $T^{*}$. However, in the intermediate-temperature range (i.e., $T_{\mathrm{c}}<T$ $\lesssim T^{*}$ ) the long-range spatial and temporal coherence in the phase of Cooper-pair wave functions (occurring for $T<T_{\mathrm{c}}$ ) is presumed to be present only up to intermediate length scales, having been disrupted on longer length scales by the presence and motion of vortex excitations. In other words, although long-range phase coherence is absent in it, the pseudogap regime is quantitatively distinguished from the conventional nonsuperconducting state by the presence of substantial, residual, local $d$-wave pairing correlations.

Several recent experimental investigations support the notion of the phase-fluctuation scenario as the origin of pseudogap phenomenology, including Refs. 14 and 15. Further steps towards an understanding of the nature of the pseudogap regime would be furnished by experimental probes that are targeted towards the question of the existence of the putative local superconducting correlations. ${ }^{16-19}$ Such probes would have the potential to discriminate between scenarios based on pairing correlations and those in which the pseudogap is due to some other mode of electronic ordering. ${ }^{4}$ The purpose of the present paper is to identify one such probe: scanning-tunneling-spectroscopy (STS) measurements of the single-particle spectral function near extended impurities in the pseudogap regime. ${ }^{20,21}$

Before explaining the nature of this probe, let us pause to recall one of its essential ingredients. It is has long been recognized $^{22-26}$ that the scattering of quasiparticles between states corresponding to differing signs of the $d$-wave pair potential leads to the existence of low-energy states. ${ }^{25,26}$ Such scattering, and hence low-energy state formation, occurs, e.g., at suitably oriented surfaces in the cuprates, leading to the observed zero-bias anomaly in the tunneling conductance. ${ }^{27}$ In the setting of impurity (rather than boundary) scattering in $d$-wave superconductors, low-energy states, which in this case are localized near the impurities, have been observed in STS experiments, ${ }^{28,29}$ and have been discussed theoretically for the case of pointlike ${ }^{30}$ and extended $^{31,32}$ impurities. We remind the reader that these states co-exist with the continuum of low-energy quasiparticle states associated with the nodes at which the $d$-wave pair potential vanishes. Distinguishing between impurity states and nodal states is straightforward because the former give a peaked contribution to the spectral function whereas the contribution from the latter vanishes linearly at zero energy. (A clear example of this is furnished by the data reported in Ref. 28.)

Returning to our main task, viz., probing the pseudogap regime for pairing correlations, we now state the central idea on which the present paper is based. Let us suppose that the pseudogap state is indeed distinguished by the presence of local (but not long range) $d$-wave pairing correlations. The lack of long-range phase coherence in such a state undermines the efficacy of conventional tests (such as the Meissner effect) for superconducting correlations. However, the low-energy quasiparticle states occurring near extended impurities are localized in space, i.e., their existence only relies on the presence of local $d$-wave pairing correlations. In con- 
sequence, these states should be only weakly affected by the destruction of long-range superconducting order that occurs at $T_{\mathrm{c}}$, and hence should "survive" the transition into the pseudogap state. Thus, if STS experiments were to reveal a sharp feature in the single-particle spectral function at lowvoltage bias, this would be evidence for the presence of local $d$-wave pairing correlations. And if such experiments were able to characterize the temperature and doping dependences of the width of this spectral feature, this would provide a characterization of the nature of these finite-range spatial and temporal correlations. Of course, the interpretation of any experiment conducted at nonzero temperature would have to contend with broadening (arising, e.g., from thermal fluctuations of the sample). In order to minimize the consequent smearing of the spectral function, which has the potential to obscure the very feature being sought, it would be preferable to examine cuprate systems having low values of $T_{\mathrm{c}}$ (e.g. heavily underdoped systems).

Why are we focusing on the case of (nonmagnetic) extended impurities as a probe of the pseudogap regime? The main reason underlying this choice is as follows. In the setting of extended impurities (as well as boundaries) in the superconducting state, it has been shown that the existence of low-energy quasiparticle states is a direct consequence of the $d$-wave nature (or, more precisely, the sign-changing nature) of the pair potential. ${ }^{31}$ Extending this reasoning to the case of extended impurities in the pseudogap regime, there the existence of low-energy states strongly depends on the presence of superconducting correlations of $d$-wave type.

We note that Kruis et al. ${ }^{33}$ have studied the density of states near a pointlike impurity in the context of a simple phenomenological picture of the pseudogap regime. In this picture, the physics of the pseudogap regime is incorporated through the hypothesis that, in the absence of the impurity, the single-particle density of states vanishes linearly at the Fermi energy. At present, the extent to which an approach based on this picture can yield information about pairing correlations in the pseudogap regime is not clear.

The present paper is organized as follows. In Sec. II we provide a framework for discussing the influence of local $d$-wave pairing correlations on quasiparticle states near extended impurities ${ }^{34}$ focusing on the single-particle spectral function. As we shall see, our expression for this spectral function will take the form of a density of states (determined at a fixed, locally phase-randomized $d$-wave pair potential) averaged over the fluctuations of the phase field. In Sec. III we develop a semiclassical scheme for computing this density of states at fixed pair potential in which we treat the long-wavelength pair-potential phase variations via perturbation theory. This scheme allows us to focus on the contribution to the density of states at low energies, which involves states arising from changes in sign (as the momentum is varied) of the local pair potential. At this point we will have obtained an expression for the spectral function, which consists of a sum of terms each associated with one classical scattering trajectory that passes through the vicinity of the impurity potential. In Sec. IV A we perform the average over the configurations of the fluctuating pair potential arrived at via local randomizations of the phase, under the assump- tion that the distribution of phase-field configurations is Gaussian. We make an approximation to the resulting expression for the spectral function that is valid for the case of phase correlations persisting beyond the Cooper-pair size, finally arriving at an expression for the spectral function near an extended impurity that consists of a Gaussian peak the linewidth of which is proportional to the typical gradient of the phase of the pair potential. In this section we also compute the linewidth of the spectral function in the pseudogap regime, doing so by assuming that the phase fluctuations are governed by the (Berezinski1-Kosterlitz-Thouless) BKT theory of the two-dimensional $X Y$ model. Finally, in Sec. V we make a numerical estimate of the linewidth by invoking the results of recent high-frequency conductivity data ${ }^{14}$ and provide some concluding remarks.

\section{MODEL OF CUPRATES WITH AN EXTENDED IMPURITY}

In this section we formulate the task of obtaining the single-particle spectral function in settings of systems of fermions interacting via some fermion-fermion coupling and also interacting with an external single-fermion potential (which could, e.g., represent an impurity potential). As our aim is to address the phase-fluctuation picture of the pseudogap regime of the cuprates, we envision following the standard field-theoretic route (see, e.g., Ref. 35) of exchanging the fermion-fermion coupling for a suitable collective quantum field $\Delta$. Thus we arrive at the following formula for the one-fermion Green function $\mathcal{G}\left(x, x^{\prime}\right)$ :

$$
\begin{gathered}
\mathcal{G}\left(x, x^{\prime}\right)=\frac{\int D \Delta^{\dagger} D \Delta e^{-S[\Delta]} G\left(x, x^{\prime} ; \Delta\right)}{\int D \Delta^{\dagger} D \Delta e^{-S[\Delta]}}, \\
\left(\begin{array}{cc}
\partial_{\tau}+\hat{h} & \hat{\Delta} \\
\hat{\Delta}^{\dagger} & \partial_{\tau}-\hat{h}
\end{array}\right) G\left(x, x^{\prime} ; \Delta\right)=-\delta\left(x-x^{\prime}\right),
\end{gathered}
$$

i.e., a Bogoliubov-de Gennes Green function for pair potential $\Delta$, suitably averaged over quantum field $\Delta$, the action for which is $S[\Delta]$. Here, $x \equiv(\mathbf{r}, \tau)$, where $\mathbf{r}$ and $\tau$ are, respectively, the spatial position (in the two-dimensional $\mathrm{CuO}_{2}$ plane) and the Matsubara time. Furthermore, $\hat{h} \equiv-\nabla^{2}-k_{F}^{2}$ $+V(\mathbf{r})$, in which $k_{F}^{2}$ is the chemical potential [i.e., $k_{F}$ $\left(\equiv 2 \pi / \lambda_{F}\right)$ is the Fermi wave vector], $V$ is the single-particle potential, and we have adopted units in which $\hbar^{2} / 2 m=1, m$ being the (effective) mass of the electrons and holes. The operator $\hat{\Delta}$ is the pair-potential (integral) operator; how it acts is specified by the nonlocal kernel $\Delta\left(x, x^{\prime}\right)$ via $[\hat{\Delta} v](x)=\int d x^{\prime} \Delta\left(x, x^{\prime}\right) v\left(x^{\prime}\right)$.

Our primary interest is in spectral function $\rho(E)$ in the pseudogap regime as well as the superconducting-topseudogap transition regime. This quantity can be obtained in the usual way from $\mathcal{G}\left(x, x^{\prime}\right)$ as follows:

$$
\rho(E) \equiv-\left.\pi^{-1} \operatorname{Im} \operatorname{Tr} \int d^{2} r \mathcal{G}\left(\mathbf{r}, \mathbf{r} ; \omega_{n}\right)\right|_{i \omega_{n} \rightarrow E+i \delta},
$$




$$
\mathcal{G}\left(\mathbf{r}, \mathbf{r}^{\prime} ; \omega_{n}\right) \equiv \int_{0}^{\beta} d \tau e^{i \omega_{n} \tau} \mathcal{G}\left(\mathbf{r}, \mathbf{r}^{\prime} ; \tau, 0\right)
$$

where $\operatorname{Tr}$ denotes a trace only in the $2 \times 2$ particle-hole space, $\beta \equiv 1 / T$ (i.e., we have chosen units in which Boltzmann's constant $k_{\mathrm{B}}=1$ ), the $\omega_{n} \equiv(2 n+1) \pi T$ (with $n$ integral) are fermionic Matsubara frequencies, and $\delta=0+$. We shall assume that the temperature is sufficiently high to validate the neglect of nonzero Matsubara-frequency modes of $\Delta$, which amounts to treating $\Delta$ as a classical (i.e., nonquantal) statistical field. Under this static condition, in which the fermion dynamics takes place in the presence of an unchanging $\Delta$ field, the spectral function $\rho(E)$ may be expressed in the form

$$
\begin{gathered}
\rho(E)=\langle\rho(E ; \Delta)\rangle, \\
\rho(E ; \Delta)=\sum_{n} \delta\left(E-E_{n}\right),
\end{gathered}
$$

where $\left\{E_{n}\right\}$ is the collection of energy eigenvalues of the following Bogoliubov-de Gennes eigenproblem in the presence of a generic classical configuration of $\Delta$

$$
\left(\begin{array}{cc}
\hat{h} & \hat{\Delta} \\
\hat{\Delta}^{\dagger} & -\hat{h}
\end{array}\right)\left(\begin{array}{l}
u \\
v
\end{array}\right)=E\left(\begin{array}{l}
u \\
v
\end{array}\right) .
$$

The notation $\langle\cdots\rangle$ denotes the aforementioned static average over $\Delta$, i.e.,

$$
\langle\mathcal{O}\rangle \equiv \frac{\int D \Delta^{\dagger} D \Delta e^{-S[\Delta]} \mathcal{O}}{\int D \Delta^{\dagger} D \Delta e^{-S[\Delta]}} .
$$

(We do not specifically indicate that this functional average is only over static configurations of $\Delta$.) Thus we have expressed the single-particle spectral function $\rho(E)$ in terms of a suitably averaged density of states $\rho(E ; \Delta)$ for a corresponding Bogoliubov-de Gennes eigenproblem.

Before proceeding with the analysis of the eigenproblem given in Eq. (4), we address the issue of the form of the pairing fluctuations that contribute dominantly to the average in Eq. (3a). This amounts to a statement about the physical picture of the pseudogap regime that we are concerned with. Now, as discussed shortly before Eq. (3a), we are considering only static configurations of $\Delta$, and therefore we shall henceforth simplify the notation by writing the pair-potential kernel $\Delta\left(x, x^{\prime}\right)$ as $\Delta\left(x, x^{\prime}\right)=\Delta\left(\mathbf{r}, \mathbf{r}^{\prime}\right)$. In addition, it is convenient to transform $\Delta\left(\mathbf{r}, \mathbf{r}^{\prime}\right)$ to relative and center-of-mass coordinates, $\boldsymbol{\rho}$ and $\mathbf{R}$,

$$
\bar{\Delta}(\boldsymbol{\rho}, \mathbf{R}) \equiv \Delta\left(\mathbf{r}, \mathbf{r}^{\prime}\right), \quad \boldsymbol{\rho} \equiv \mathbf{r}-\mathbf{r}^{\prime}, \quad 2 \mathbf{R} \equiv \mathbf{r}+\mathbf{r}^{\prime} .
$$

It is then convenient to introduce the Fourier transform of $\bar{\Delta}$ with respect to the relative coordinate $\boldsymbol{\rho}$, viz., $\bar{\Delta}(\mathbf{k}, \mathbf{R})$,

$$
\bar{\Delta}(\mathbf{k}, \mathbf{R}) \equiv \int d^{2} \rho e^{-i \mathbf{k} \cdot \boldsymbol{\rho}} \bar{\Delta}(\boldsymbol{\rho}, \mathbf{R}),
$$

thus obtaining a pair potential at center-of-mass position $\mathbf{R}$ and relative momentum $\mathbf{k}$.

As mentioned in Sec. I, the scenario for the pseudogap regime with which we are concerned is based on the dominance of configurations of $\bar{\Delta}(\mathbf{k}, \mathbf{R})$ of the form

$$
\bar{\Delta}(\mathbf{k}, \mathbf{R})=\bar{\Delta}_{0}(\mathbf{k}, \mathbf{R}) e^{i \vartheta(\mathbf{R})}
$$

Here, the nonfluctuating factor $\bar{\Delta}_{0}(\mathbf{k}, \mathbf{R})$ is taken to have $d$-wave form (and can, therefore, be taken to be real). ${ }^{36}$ The fluctuating factor $\exp i \vartheta(\mathbf{R})$ varies slowly with $\mathbf{R}$. By assuming this form for $\bar{\Delta}(\mathbf{k}, \mathbf{R})$ we are adopting the physical picture of the state as being one in which there is no long-range superconducting order, but there are local $d$-wave superconducting correlations, embodied in $\bar{\Delta}_{0}(\mathbf{k}, \mathbf{R}){ }^{37}$

In the following section, we obtain $\rho(E ; \Delta)$ by making use of an elaboration of Andreev's semiclassical approach ${ }^{38}$ to the Bogoliubov-de Gennes eigenproblem. This elaboration is appropriate for the setting at hand, viz., one in which there is a strong single-particle potential. This scheme was used in Ref. 31 in order to address the low-energy density of states near an extended impurity in a $d$-wave superconducting state having a negligibly fluctuating, well-formed condensate. In contrast, our focus here is on situations in which the fluctuations in the amplitude of $\Delta$ are small, but the phase of $\Delta$ is strongly fluctuating. Thus, although there is local pairing the system does does not exhibit long-range order.

\section{SEMICLASSICAL APPROACH TO THE BOGOLIUBOV-DE GENNES EIGENPROBLEM}

In Sec. II we showed how the single-particle spectral function $\rho(E)$ can be expressed as a density of states $\rho(E ; \Delta)$ for the Bogoliubov-de Gennes eigenproblem at arbitrary pair potential $\Delta$, averaged over $\Delta$ with a suitable weight, provided $\Delta$ can be treated in the static approximation. To make progress with this Bogoliubov-de Gennes eigenproblem we invoke, in the present section, a semiclassical approximation under which Eq. (4) reduces to a family of one-dimensional eigenproblems labeled by the classical scattering trajectories of a particle in the presence of the single-particle potential $V(\mathbf{r})$. This approximation scheme, which was developed in Ref. 31 , is valid provided that $k_{F}^{2}$ $\gg\left\{\Delta_{0}, E\right\}$ for physically relevant configurations of $\Delta$ (where $\Delta_{0}$ is the magnitude of $\Delta$ ), and provided that $V(\mathbf{r})$ and relevant configurations of $\Delta$ vary slowly on the scale of the Fermi wavelength $\lambda_{\mathrm{F}}$.

We now turn to the family of one-dimensional eigenproblems arising in our semiclassical scheme (see Fig. 1). Following Ref. 31, it is straightforward to determine that the emerging trajectory-dependent eigenproblem has the form

$$
\hat{H}\left(\begin{array}{l}
\bar{u} \\
\bar{v}
\end{array}\right)=E\left(\begin{array}{l}
\bar{u} \\
\bar{v}
\end{array}\right)
$$




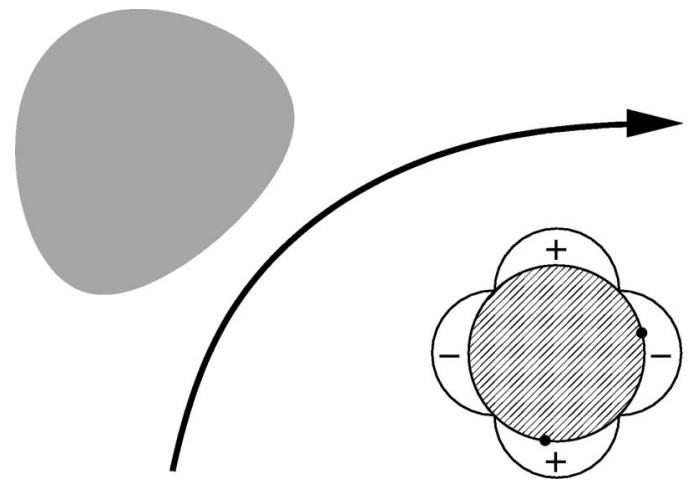

FIG. 1. Schematic illustration of a classical trajectory for a quasiparticle scattering from an extended impurity. Also shown is a depiction of the background $d$-wave pair potential; dots indicate incoming and outgoing quasiparticle momenta.

$$
\begin{gathered}
\hat{H} \equiv\left(\begin{array}{cc}
-2 i k_{F} \partial_{s} & \Delta_{0}(s) \exp i \vartheta(s) \\
\Delta_{0}(s) \exp -i \vartheta(s) & 2 i k_{F} \partial_{s}
\end{array}\right), \\
\Delta_{0}(s) \simeq \bar{\Delta}_{0}\left[k_{F} \partial_{s} \mathbf{x}_{\mathrm{c}}(s), \mathbf{x}_{\mathrm{c}}(s)\right] .
\end{gathered}
$$

Here, $\vartheta(s) \simeq \vartheta\left(\mathbf{x}_{\mathrm{c}}(s)\right)$, and the parameter $s$ measures the position along a particular classical trajectory $\mathbf{x}_{\mathrm{c}}(s)$, the latter obeying the Newton equation

$$
k_{F}^{2} \partial_{s}^{2} \mathbf{x}_{\mathrm{c}}(s)=-\boldsymbol{\nabla} V\left(\mathbf{x}_{\mathrm{c}}(s)\right) .
$$

Each such classical trajectory (and associated eigenproblem) is labeled by an impact parameter $b$ and an incoming momentum direction $\mathbf{n}$.

Our next task is to obtain the low-energy eigenvalues associated with Eq. (9a) for the case of a $d$-wave pair potential subject to generic spatial phase variations, i.e., Eq. (8). To do this, we perform a local unitary transformation of $\hat{H}$, i.e., $\hat{H} \rightarrow U \hat{H} U^{\dagger}$, where

$$
U(s) \equiv\left(\begin{array}{cc}
e^{-i \vartheta(s) / 2} & 0 \\
0 & e^{i \vartheta(s) / 2}
\end{array}\right),
$$

and thus our eigenproblem acquires the form

$$
\begin{gathered}
\left(\hat{H}_{0}+\hat{H}_{1}\right)\left(\begin{array}{c}
\bar{u} \\
\bar{v}
\end{array}\right)=E\left(\begin{array}{c}
\bar{u} \\
\bar{v}
\end{array}\right), \\
\hat{H}_{0}(s) \equiv\left(\begin{array}{cc}
-2 i k_{F} \partial_{s} & \Delta_{0}(s) \\
\Delta_{0}(s) & 2 i k_{F} \partial_{s}
\end{array}\right), \\
\hat{H}_{1}(s) \equiv\left(\begin{array}{cc}
k_{F} \partial_{s} \vartheta & 0 \\
0 & k_{F} \partial_{s} \vartheta
\end{array}\right) .
\end{gathered}
$$

The Hamiltonian for this eigenproblem now consists of a term arising from the underlying $d$-wave pair potential (i.e. $\hat{H}_{0}$ ) as well as a term that contains all the phase-variation information (i.e., $\hat{H}_{1}$ ). Our strategy is to treat $\hat{H}_{1}$ within perturbation theory, the starting point for which is the identification of the eigenstates of $\hat{H}_{0}$. As our purpose is to address low-energy states, it is adequate for us to focus on the zeroenergy eigenstate of $\hat{H}_{0}$, if any there be. As we shall discuss below, such states are guaranteed to arise for certain classical trajectories $\mathbf{x}_{\mathrm{c}}(s) .{ }^{31,39}$ The condition for the existence of such a state is determined by the asymptotic properties of $\Delta_{0}(s)$ : If $\Delta_{0}(s)$ changes sign an odd number of times along an entire trajectory then the Hamiltonian $\hat{H}_{0}$ associated with that trajectory has precisely one zero-energy eigenstate. Said more formally, if $\Delta_{ \pm} \equiv \lim _{s \rightarrow \pm \infty} \Delta_{0}(s)$ then the condition for the existence of the zero-energy state is that $\Delta_{+} \Delta_{-}<0$. The explicit form of this eigenstate is

$$
\begin{gathered}
\psi_{ \pm}(s)=\frac{1}{\sqrt{2}}\left(\begin{array}{c}
\varphi_{ \pm}(s) \\
\pm i \varphi_{ \pm}(s)
\end{array}\right), \\
\varphi_{ \pm}(s) \equiv \alpha_{ \pm} \exp \pm\left(2 k_{F}\right)^{-1} \int^{s} d s^{\prime} \Delta_{0}\left(s^{\prime}\right),
\end{gathered}
$$

where $\psi_{+}$corresponds to the case $\Delta_{+}<0$ (so that $\Delta_{-}>0$ ) and $\psi_{-}$corresponds to the case $\Delta_{+}>0$ (so that $\left.\Delta_{-}<0\right)$. The prefactors $\alpha_{ \pm}$are normalization factors, chosen so that $\int_{-\infty}^{\infty} \varphi_{ \pm}(s)^{2} d s=1$.

Let us emphasize some of the important features of these zero-energy states that hold provided the scatterer creates only trajectories with at most a single sign change in the pair potential. ${ }^{40}$ First, the presence or absence of these states depends only on the properties of the $d$-wave pair potential far from any impurity, and is insensitive to any amplitude variations of the $d$-wave pair potential that might occur in the vicinity of this impurity. Second, the wave functions $\psi_{ \pm}(s)$ exhibit exponential decay away from the impurity with a decay constant of order $k_{F} /\left|\Delta_{ \pm}\right|$, which is proportional to the BCS correlation length. As this length scale is known to be short in the cuprates, these states are indeed an extremely local probe of local superconducting correlations.

In the absence of phase fluctuations (i.e., in the pure $d$-wave superconductor), these zero-energy eigenstates lead to a sharp peak in the density of states at low energies. ${ }^{31}$ To access the impact of order-parameter phase variations on this peak, we include the effect of $\hat{H}_{1}$ on the zero-energy state $\psi_{ \pm}^{\dagger}(s)$ within quantum-mechanical perturbation theory. Thus, the shift in energy of the previously zero-energy eigenstate associated with a particular trajectory $\mathbf{x}_{\mathrm{c}}(s)$ (parametrized by $\mathbf{n}$ and $b$ ) is given by

$$
\begin{aligned}
\boldsymbol{\epsilon}(\mathbf{n}, b) & \equiv \int d s \psi_{ \pm}^{\dagger}(s) \hat{H}_{1}(s) \psi_{ \pm}(s), \\
& =k_{F} \int d s \partial_{s} \vartheta(s) \varphi_{ \pm}(s)^{2},
\end{aligned}
$$

where we have used Eq. (13a) to get from Eq. (14a) to Eq. (14b). The spectral function at fixed pair potential $\rho(E, \Delta)$ consists of contributions from all trajectories $\mathbf{x}_{\mathrm{c}}(s)$. As we are restricting our attention to low energies, it is sufficient to consider only trajectories for which $\Delta_{+} \Delta_{-}<0$ (i.e., those for which $\hat{H}_{0}$ has a zero-energy state). The contribution to the 
low-energy spectral function at constant pair potential due to the perturbed zero-energy states is given by

$\rho(E, \Delta) \simeq k_{F} \int \frac{d \mathbf{n}}{2 \pi} \int d b \delta(E-\epsilon(\mathbf{n}, b))\left(1-\operatorname{sgn} \Delta_{+} \operatorname{sgn} \Delta_{-}\right)$,

where the factor $\left(1-\operatorname{sgn} \Delta_{+} \operatorname{sgn} \Delta_{-}\right)$ensures that only trajectories that satisfy $\Delta_{+} \Delta_{-}<0$ [i.e., exhibit an asymptotic sign change in $\left.\Delta_{0}(s)\right]$ contribute.

It is important to emphasize that Eq. (15) only includes the contribution from the perturbed zero-energy states, and that there will be additional, smaller contributions to the density of states at low energies. Sources of these include remnants of the near-nodal quasiparticles states that exist in pure, $d$-wave superconductors at arbitrarily low energies.

\section{AVERAGING OVER PHASE FLUCTUATIONS}

In Sec. III we obtained an expression for the Bogoliubov-de Gennes density of states $\rho(E, \Delta)$ in the presence of an extended impurity for the case of a pair-potential with local $d$-wave character and specific realization of the spatially varying phase $\vartheta(\mathbf{r})$; see Eq. (15). In the present section, we calculate the spectral function $\rho(E)$ in the pseudogap regime by averaging $\rho(E, \Delta)$ over suitable configurations of $\vartheta(\mathbf{r})$, i.e., by inserting Eq. (15) into Eq. (3a). We remind the reader that our scheme for computing $\rho(E)$ applies to settings in which the temperature is high enough that we may treat the phase fluctuations classically, as discussed after Eq. (2b).

\section{A. Gaussian model for phase fluctuations}

In order to evaluate the average over configurations of the pair potential in Eq. (3a) explicitly, we need a model for the weight of the various configurations. For the sake of simplicity, we choose the phase-field configurations to have a (zero mean) Gaussian distribution characterized by the correlator $\left\langle\nabla_{\alpha} \vartheta(\mathbf{r}) \nabla_{\beta} \vartheta\left(\mathbf{r}^{\prime}\right)\right\rangle$. In terms of this correlator, it is straightforward to show that the spectral function $\rho(E)$ is a superposition of Gaussian distributions, one associated with each classical trajectory on which $\Delta_{0}$ changes sign,

$$
\begin{aligned}
& \rho(E) \simeq k_{F} \int \frac{d \mathbf{n}}{2 \pi} \int d b \frac{\exp \left(-E^{2} / 2\left\langle\epsilon(\mathbf{n}, b)^{2}\right\rangle\right)}{\sqrt{2 \pi\left\langle\epsilon^{2}\right\rangle}} \\
& \times\left(1-\operatorname{sgn} \Delta_{+} \operatorname{sgn} \Delta_{-}\right), \\
&\left\langle\boldsymbol{\epsilon}(\mathbf{n}, b)^{2}\right\rangle \equiv k_{F}^{2} \int d s \int d s^{\prime} \varphi_{ \pm}^{2}(s) \varphi_{ \pm}^{2}\left(s^{\prime}\right)\left\langle\partial_{s} \vartheta(s) \partial_{s^{\prime}} \vartheta\left(s^{\prime}\right)\right\rangle .
\end{aligned}
$$

Here, the width $\left\langle\epsilon(\mathbf{n}, b)^{2}\right\rangle$ of each Gaussian contribution is sensitive to the degree to which local superconducting correlations have been disrupted, as can be seen by the presence of the phase-phase correlator $\left\langle\partial_{s} \vartheta(s) \partial_{s^{\prime}} \vartheta\left(s^{\prime}\right)\right\rangle$ in Eq. (16b). Our next task, then, is to evaluate the integrals over the parameters $s$ and $s^{\prime}$ in Eq. (16b) in order to determine the width $\left\langle\epsilon(\mathbf{n}, b)^{2}\right\rangle$ associated with each trajectory $(\mathbf{n}, b)$. In the following section, we carry out this evaluation within an approximation that is valid for the case of long-wavelength phase fluctuations.

\section{B. Approximate evaluation of trajectory integrals}

In Sec. IV A, we obtained the expression (16a) for the spectral function $\rho(E)$ near an extended impurity in the pseudogap regime. In the present section, we make an approximation to our expression for $\rho(E)$ that makes use of the local nature of the low-energy states, as well as the longwavelength nature of the pair-potential phase variations (appropriate for $T \gtrsim T_{\mathrm{c}}$ ). By local we mean that the wave functions $\varphi_{ \pm}(s)$ exhibit exponential decay over a length scale $\xi$ $\sim k_{F} /\left|\Delta_{ \pm}\right|$(where $\Delta_{0}$ is the bulk $d$-wave pair potential), i.e., the BCS correlation length; this can be seen by examining Eq. (13b); see Ref. 41. In the cuprate superconductors, this length scale is expected to be much shorter than the length scale $\xi_{\vartheta}$ for typical pair-potential phase variations (i.e., the intervortex spacing). Thus one has a separation of length scales: $\xi_{\vartheta}>\xi$. (Such a separation is a natural ingredient of the phase-fluctuation picture of the pseudogap regime because for intervortex spacings on the order of $\xi$ the conventional meaning of local Cooper pairs breaks down.)

To make the approximation to our expression for $\rho(E)$, consider the integrations over the trajectory parameters $s$ and $s^{\prime}$ in Eq. (16b). Now, the correlation function $\left\langle\partial_{s} \vartheta(s) \partial_{s^{\prime}} \vartheta\left(s^{\prime}\right)\right\rangle$ varies appreciably only over length scales on the order of $\xi_{\vartheta}$ or longer, whereas the wave functions $\varphi_{ \pm}$ decay exponentially, as mentioned, on the length scale $\bar{\xi}$. Hence, one can make an asymptotic approximation to the $s$ and $s^{\prime}$ integrations, which amounts to pulling the correlator out of the integrals. Thus, owing to the normalization of $\varphi_{ \pm}$, we have

$$
\left\langle\epsilon^{2}\right\rangle \simeq k_{F}^{2}\left\langle\left.\partial_{s} \vartheta(s)\right|_{s=0} ^{2}\right\rangle,
$$

independent of $\mathbf{n}$ and $b$.

Next, we turn to the interpretation of the quantity $\left\langle\left.\partial_{s} \vartheta(s)\right|_{s=0} ^{2}\right\rangle$. The derivative of the phase along a particular trajectory is given by

$$
\partial_{s} \vartheta(s)=\left.\partial_{s} \mathbf{x}_{\mathrm{c}}(s) \cdot \boldsymbol{\nabla} \vartheta(\mathbf{x})\right|_{\mathbf{x}=\mathbf{x}_{\mathrm{c}}(s)},
$$

By inserting Eq. (18) into Eq. (17), we see that the correlator of interest is $\left\langle\nabla_{\alpha} \vartheta(\mathbf{r}) \nabla_{\beta} \vartheta(\mathbf{r})\right\rangle$ which, by spatial isotropy, has the form $\delta_{\alpha \beta}\left\langle|\nabla \vartheta(\mathbf{r})|^{2}\right\rangle / 2$. According to the phasefluctuation scenario, vortex excitations are the dominant mechanism for generating phase gradients.

How does this information about the phase correlations translate into information about the spectral function? By noting that trajectories $\mathbf{x}_{\mathrm{c}}(s)$ involving sign changes in the $d$-wave pair potential obey $\left|\partial_{s} \mathbf{x}_{\mathrm{c}}\right| \sim 1$ and using the aforementioned phase-gradient correlator, we arrive at the width

$$
\left\langle\epsilon^{2}\right\rangle \sim k_{F}^{2}\left\langle|\nabla \vartheta(\mathbf{r})|^{2}\right\rangle / 2
$$

and, hence, our final expression for the spectral function $\rho(E)$, 


$$
\begin{aligned}
\rho(E) \simeq & \frac{\exp \left(-E^{2} / 2\left\langle\epsilon^{2}\right\rangle\right)}{\sqrt{2 \pi\left\langle\epsilon^{2}\right\rangle}} k_{F} \int \frac{d \mathbf{n}}{2 \pi} \\
& \times \int d b\left(1-\operatorname{sgn} \Delta_{+} \operatorname{sgn} \Delta_{-}\right) .
\end{aligned}
$$

The first factor is a Gaussian in the energy $E$ with a linewidth $\left\langle\epsilon^{2}\right\rangle^{1 / 2}$ associated with the rms fluctuations in the phase gradient. The second factor (i.e. the integral over the impact parameter $b$ and the incoming momentum direction $\mathbf{n}$ ) determines the scale for $\rho(E)$, essentially by counting the number of sign-changing trajectories and it is expected to be only weakly temperature dependent. In the following section, we compute the linewidth within a Berezinskiı̆-KosterlitzThouless-like model of phase fluctuations near $T_{\mathrm{c}}$.

\section{Linewidth near the superconducting phase boundary}

To make further progress, we now attempt to calculate the spectral function linewidth due to the phase fluctuations accompanying the destruction of superconducting order in the neighborhood of $T_{\mathrm{c}}$. We shall do this by choosing a particular weight for the phase fluctuations, viz., that associated with the two-dimensional $X Y$ model. ${ }^{42-44}$ Our analysis is reminiscent of that due to Franz and Millis ${ }^{11}$ who addressed the bulk single-particle spectral function in the pseudogap regime. In the present context, this analysis is provided solely for illustrative purposes, and is meant only to provide a rough estimate of the linewidth.

Let us consider the $X Y$-model action

$$
S[\Delta]=\frac{K}{2} \int d^{2} r|\nabla \vartheta|^{2},
$$

where $K \equiv \rho_{s}(T) / T$ in which $\rho_{s}(T)$ is the temperaturedependent superfluid density. ${ }^{45,46}$ Note that we are not suggesting that the true critical fluctuations of the cuprate superconductors necessarily lie in the universality class of the two-dimensional $X Y$ model, but simply that the intermediate length-scale fluctuations proposed as leading to pseudogap phenomena may adequately be modeled by Eq. (21). The form for $\left\langle|\boldsymbol{\nabla} \vartheta(\mathbf{r})|^{2}\right\rangle$ for the $X Y$ model may be calculated following the Debye-Hückel-type analysis of Halperin and Nelson, ${ }^{47}$ giving

$$
\left\langle|\nabla \vartheta(\mathbf{r})|^{2}\right\rangle \simeq \frac{2}{\pi \xi_{\vartheta}^{2} K^{*}} \ln \Lambda \xi_{\vartheta},
$$

in which $K^{*}$ is the short-length-scale stiffness (obtained using the Kosterlitz renormalization-group equations), $\Lambda$ is a short-distance cutoff, and $\xi_{\vartheta}$ is a length scale characterizing the typical intervortex spacing. The principal temperature dependence in Eq. (22) arises via $\xi_{\vartheta}$ : near $T_{\mathrm{BKT}}$ [i.e., the transition temperature of the model (21), which is expected to lie not far below $\left.T_{\mathrm{c}}\right] \xi_{\vartheta}$ is proportional to $\exp \sqrt{\Theta /\left(T-T_{\mathrm{BKT}}\right)}$, where $\Theta$ is a constant of order unity (which we do not try to calculate in detail).

The proliferation of unbound vortex excitations upon warming through the BKT transition is reflected (see Fig. 2)

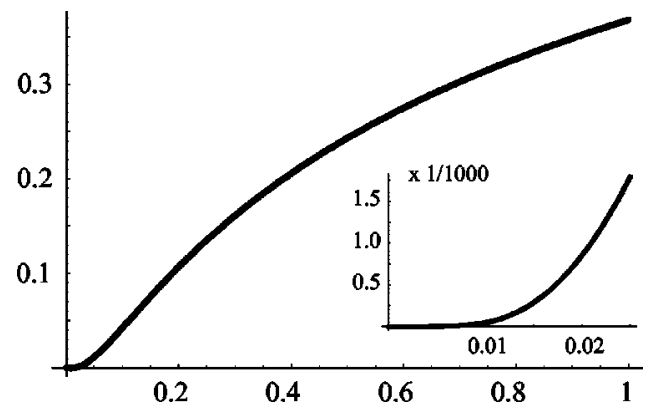

FIG. 2. Spectral linewidth versus $\left(T-T_{\mathrm{BKT}}\right) / \Theta$. Inset: Immediate vicinity of the transition.

by this strong diminution of $\xi_{\vartheta}$ and causes a concomitant dramatic increase in the linewidth of the spectral function

$$
\left\langle\epsilon^{2}\right\rangle^{1 / 2} \propto \exp \left[-\sqrt{\Theta /\left(T-T_{\mathrm{BKT}}\right)}\right] .
$$

Observation of a fluctuation-broadened peak in the spectral function, e.g., via STS measurements, would provide striking evidence in support of the phase-fluctuation scenario. Moreover, the temperature dependence of the linewidth would, e.g., provide access to the details of the vortex-unbinding transition.

\section{NUMERICAL ESTIMATE OF THE LINEWIDTH; CONCLUDING REMARKS}

In the preceding section we saw how one could estimate the temperature dependence of the linewidth of the spectral function near the superconducting transition temperature $T_{\mathrm{c}}$. In the present section we make a rough numerical estimate of this linewidth at one particular temperature in the pseudogap regime by appealing to the data obtained in the highfrequency conductivity experiments of Corson et al. ${ }^{14}$ on $\mathrm{Bi}_{2} \mathrm{Sr}_{2} \mathrm{CaCu}_{2} \mathrm{O}_{8}+\delta$. We shall be specifically concerned with the interpretation of these data inasmuch as they provide access to the characteristic vortex density.

Until now we have been working with a system of units in which $\hbar^{2} / 2 m=1$. Restoring conventional units in Eq. (19) gives for the linewidth

$$
\left\langle\epsilon^{2}\right\rangle^{1 / 2} \sim \frac{\hbar^{2}}{2 m} k_{F}\left\langle|\nabla \vartheta(\mathbf{r})|^{2} / 2\right\rangle^{1 / 2} .
$$

To estimate this width, we turn to the experiments of Corson et al. and its analysis by Corson et al., which is based on the notion that, at sufficiently high frequencies, the conductivity probes short-length-scale pairing correlations, and leads to an estimate for the characteristic density of free vortices $n_{\mathrm{f}}$. Assuming that it is vortex excitations that lead to phase fluctuations, one expects that, up to a constant of order unity, $\left\langle|\boldsymbol{\nabla} \vartheta(\mathbf{r})|^{2}\right\rangle \simeq n_{\mathrm{f}}$.

Now, Corson et al. obtain values of $n_{f}$ that are on the order of $0.003 a_{\mathrm{vc}}$ (for $T \sim 75 \mathrm{~K}$ ), where $a_{\mathrm{vc}}$ is the area of the core of a vortex. If we take the vortex core to be a disk of radius $\xi \simeq 1 \mathrm{~nm}$, this leads to the value $n_{\mathrm{f}} \sim 10^{3} \mu \mathrm{m}^{-2}$. Then, using the order-of-magnitude estimate $k_{F} \simeq 1 \mathrm{~nm}^{-1}$, Eq. (24) gives $\left\langle\epsilon^{2}\right\rangle^{1 / 2} \simeq 9 \mathrm{meV}$. 
The value of this estimate is that it shows that, for at least one cuprate material, there is a temperature at which the free-vortex density is small enough that phase fluctuations only weakly perturb the energies of the quasiparticle states. Hence, the linewidth arising from perturbed zero-energy states can be rather smaller than the scale of the superconducting energy gap (and hence small enough to justify the our picture of perturbed zero modes) but be large enough to be resolvable in STS measurements, such as those of Yazdani et al. ${ }^{28}$ Larger densities of free vortices, and hence large linewidths, would result from working at higher temperatures. Moreover, near $T_{\mathrm{c}}$ the vortex density is expected to show a very strong dependence on temperature, which should confer a strong temperature dependence on the linewidth. Of course, if conducted at the temperature of the experiments of Corson et al., thermal broadening would complicate the task of accessing the intrinsic linewidth (i.e., the linewidth due to phase fluctuations); recall that $10 \mathrm{~K}$ is equivalent to $1 \mathrm{meV}$.
Thus, as emphasized in Sec. I, one should consider performing experiments on materials having a lower $T_{\mathrm{c}}$, so that the pseudogap regime can be explored at temperatures at which thermal broadening is less significant. These considerations indicate that it is at least conceivable that STS experiments near extended scatterers could provide a sharp test of the phase fluctuation scenario for the pseudogap regime.

\section{ACKNOWLEDGMENTS}

It is a pleasure to thank Erich Mueller for discussions. This work was supported by the U.S. Department of Energy, Division of Materials Sciences under Award No. DEFG0296ER45439, through the Materials Research Laboratory at the University of Illinois at Urbana-Champaign (D.E.S., I.A., P.M.G., A.Y.), by NSF Grant No. DMR99-75187 (P.M.G), and by Grant No. NSF DMR98-75565 (A.Y.)
*Electronic address: d-sheehy@uiuc.edu

†Electronic address: adagidel@uiuc.edu

†Electronic address: goldbart@uiuc.edu

${ }^{\S}$ Electronic address: ayazdani@uiuc.edu

${ }^{1}$ A. G. Loeser, Z.-X. Shen, D. S. Dessau, D. S. Marshall, C. H. Park, P. Fournier, and A. Kapitulnik, Science 273, 325 (1996).

${ }^{2}$ H. Ding, T. Yokoya, J. C. Campuzano, T. Takahashi, M. Randeria, M. R. Norman, T. Mochiku, K. Kadowaki, and J. Giapintzakis, Nature (London) 382, 51 (1996).

${ }^{3}$ Ch. Renner, B. Revaz, J.-Y. Genoud, K. Kadowaki, and $\emptyset$. Fischer, Phys. Rev. Lett. 80, 149 (1998).

${ }^{4}$ Among these are SO(5) (Ref. 5), SU(2) slave boson (Ref. 6), pre-formed pairs (Ref. 7), antiferromagnetic spin fluctuations (Ref. 8), and $d$-density wave (Ref. 9), to give a nonexhaustive list.

${ }^{5}$ S.-C. Zhang, Science 275, 1089 (1997).

${ }^{6}$ X.-G. Wen and P. A. Lee, Phys. Rev. Lett. 76, 503 (1996).

${ }^{7}$ J. Maly, K. Levin, and D. Z. Liu, Phys. Rev. B 54, R15 657 (1996); B. Jankó, J. Maly, and K. Levin, ibid. 56, R11 407 (1997).

${ }^{8}$ J. Schmalian, D. Pines, and B. Stojkovic, Phys. Rev. Lett. 80, 3839 (1998).

${ }^{9}$ S. Chakravarty, R. B. Laughlin, D. K. Morr, and C. Nayak, Phys. Rev. B 63, 094503 (2001)

${ }^{10}$ V. Emery and S. A. Kivelson, Nature (London) 374, 434 (1995).

${ }^{11}$ M. Franz and A. J. Millis, Phys. Rev. B 58, 14572 (1998).

${ }^{12}$ H.-J. Kwon and A. T. Dorsey, Phys. Rev. B 59, 6438 (1999).

${ }^{13}$ For a review, see V. M. Loktev, R. M. Quick, and S. Sharapov, Phys. Rep. 349, 1 (2001).

${ }^{14}$ J. Corson, R. Mallozzi, J. Orenstein, J. N. Eckstein, and I. Bozovic, Nature (London) 398, 221 (1999).

${ }^{15}$ Z. A. Xu, N. P. Ong, Y. Wang, T. Kakeshita, and S. Uchida, Nature (London) 406, 486 (2000).

${ }^{16}$ B. Jankó, I. Kosztin, K. Levin, M. R. Norman, and D. J. Scalapino, Phys. Rev. Lett. 82, 4304 (1999).

${ }^{17}$ H.-Y. Choi, Y. Bang, and D. K. Campbell, Phys. Rev. B 61, 9748 (2000).

${ }^{18}$ D. E. Sheehy, P. M. Goldbart, J. Schmalian, and A. Yazdani, Phys. Rev. B 62, 4105 (2000).
${ }^{19}$ I. Martin and A. Balatsky, Phys. Rev. B 62, R6124 (2000)

${ }^{20}$ By extended impurities we mean static, localized variations in the electrochemical potential having a spatial extent that is large compared to the Fermi wavelength $\lambda_{F}$. As possible origins of extended impurities we have in mind the deposits of $\mathrm{Au}$ atoms introduced in the experiments reported in Ref. 28. The degree to which the native defects examined in the experiments reported in Ref. 29 can be regarded as extended impurities (and thus be amenable to the approach of the present paper) is less clear. We note that the subject of states associated with extended impurities in the setting of magnetic crystals have been surveyed in Ref. 21

${ }^{21}$ M. A. Ivanov, V. M. Loktev, and Yu. G. Pogorelov, Phys. Rep. 153, 209 (1987)

${ }^{22}$ C.-R. Hu, Phys. Rev. Lett. 72, 1526 (1994).

${ }^{23}$ L. J. Buchholtz, M. Palumbo, D. Rainer, and J. A. Sauls, J. Low Temp. Phys. 101, 1079 (1995); 101, 1099 (1995).

${ }^{24}$ M. Fogelström, D. Rainer, and J. A. Sauls, Phys. Rev. Lett. 79, 281 (1997).

${ }^{25}$ In the context of scattering due to pointlike impurities (Ref. 30), the density of states possesses low-energy resonances, the energies of which move to zero energy as the strength of the impurity potential is increased. Related work has been reported by Pogorelov (Ref. 26).

${ }^{26}$ Yu. Pogorelov, Solid State Commun. 95, 245 (1995).

${ }^{27}$ M. Covington, R. Scheuerer, K. Bloom, and L. H. Greene, Appl. Phys. Lett. 68, 1717 (1996); M. Covington, M. Aprili, E. Paraoanu, L. H. Greene, F. Xu, J. Zhu, and C. A. Mirkin, Phys. Rev. Lett. 79, 277 (1997).

${ }^{28}$ A. Yazdani, C. M. Howald, C. P. Lutz, A. Kapitulnik, and D. M. Eigler, Phys. Rev. Lett. 83, 176 (1999).

${ }^{29}$ E. Hudson, S. H. Pan, A. K. Gupta, K.-W. Ng, and J. C. Davis, Science 285, 88 (1999)

${ }^{30}$ A. V. Balatsky, M. I. Salkola, and A. Rosengren, Phys. Rev. B 51, 15547 (1995); M. I. Salkola, A. V. Balatsky, and D. J. Scalapino, Phys. Rev. Lett. 77, 1841 (1996).

${ }^{31}$ I. Adagideli, P. M. Goldbart, A. Shnirman, and A. Yazdani, Phys. Rev. Lett. 83, 5571 (1999).

${ }^{32}$ The analysis of low-energy quasiparticle states reported in Ref. 
31 is based on an application of Witten's supersymmetric quantum mechanics (Ref. 39).

${ }^{33}$ H. V. Kruis, I. Martin, and A. V. Balatsky cond-mat/0008349 (unpublished).

${ }^{34}$ Among the virtues of the setting of extended (rather than pointlike) impurities are: (i) the robustness of the low-energy states, by which we mean the insensitivity of their energies to spatial variations in the modulus of the $d$-wave pair potential; and (ii) the presence of low-energy states are signatures of superconductivity, specifically with sign changes in the pair-potential.

${ }^{35}$ H. Kleinert, Fortschr. Phys. 26, 565 (1978).

${ }^{36}$ Although $\Delta_{0}(\mathbf{k}, \mathbf{R})$ is expected to be independent of $\mathbf{R}$ away from the impurity (i.e., in the bulk), near the impurity pair-breaking processes may suppress the pair-potential amplitude leading to $\mathbf{R}$ dependence.

${ }^{37}$ Note that we are ignoring broader classes of fluctuations, such as those that would take $\bar{\Delta}(\mathbf{k}, \mathbf{R})$ out of the space of $\mathrm{d}_{x^{2}-y^{2}}$ states. For example, one can envision the situation in which phasefluctuating $d$-wave order is augmented by fluctuating local $s$-wave order. Additional classes of fluctuations, such as this, can readily be incorporated by the scheme discussed in the present paper, and would lead to further broadening of the singleparticle spectral function.

${ }^{38}$ A. F. Andreev, Zh. Éksp. Teor. Fiz. 46, 1823 (1964) [Sov. Phys. JETP 19, 1228 (1964)].

${ }^{39}$ E. Witten, Nucl. Phys. B 188, 513 (1981).

${ }^{40}$ The caveat with regard to our focusing on scatterers that create trajectories having at most one sign change in the pair potential is meant to eliminate from consideration various very lowenergy states associated with trajectories involving an even number of multiple sign changes of the pair potential. In particular, we have in mind the following scenario: Imagine a system consisting of two extended impurities. In such a system, there exist trajectories that scatter twice, once from each impurity. Among these, of particular interest are the trajectories that encounter two sign changes, e.g., one can imagine a trajectory that scatters off the first impurity from the positive lobe of the $d$-wave order parameter to the negative lobe and then off the second impurity from the negative lobe back to the positive lobe. As such a trajectory has no net sign change there will be no zero-energy state. If, however, the impurities are far apart, one can imagine that the supersymmetry analysis applied to each state individually (i.e., ignoring the other impurity, and thus giving a zero-energy state for each) would be correct, up to an exponentially small tunneling correction, indicating that such a doubly scattering trajectory would provide two states, both very close indeed to zero energy.

${ }^{41}$ Of course, this exponential decay breaks down for trajectories in which at least one of the asympototic directions (incoming or outgoing) tends to a nodal direction. Such trajectories comprise a relatively small fraction of the trajectories and will, henceforth, be neglected.

${ }^{42}$ L. Berezinskiǔ, Zh. Éksp. Teor. Fiz 59, 907 (1970) [Sov. Phys. JETP 32, 493 (1971)].

${ }^{43}$ J. M. Kosterlitz and D. J. Thouless, J. Phys. C 6, 1181 (1973); J. M. Kosterlitz, ibid. 7, 1046 (1974).

${ }^{44}$ For a discussion of the $X Y$ model see, e.g., P. M. Chaikin and T. C. Lubensky, Principles of Condensed Matter Physics (Cambridge University Press, Cambridge 1995), Chap. 9.

${ }^{45}$ For a discussion of phase-fluctuation models in $d$-wave superconductors see, e.g., Ref. 46.

${ }^{46}$ A. Paramekanti, M. Randeria, T. V. Ramakrishnan, and S. S. Mandal, Phys. Rev. B 62, 6786 (2000).

${ }^{47}$ B. I. Halperin and David R. Nelson, J. Low Temp. Phys. 36, 599 (1979). 